\title{
OPERACIONALIZAÇÃO DO SISTEMA DE VIGILÂNCIA ALIMENTAR E NUTRICIONAL (SISVAN) NO MUNICÍPIO DE BALNEÁRIO PIÇARRAS: RELATO DE EXPERIÊNCIA
}

\section{Operationalization of the Food and Nutrition Surveillance System (SISVAN) in the municipality of Balneário Piçarras: an experience report}

\section{Operacionalización del Sistema de Vigilancia Alimentaria y Nutricional (SISVAN) en el municipio de Balneario Piçarras: relato de experiencia}

\author{
Éllen Luzia Soares \\ Universidade do Vale do Itajaí - UNIVALI - Itajaí (SC) - Brasil \\ Tatiana Mezadri \\ Universidade do Vale do Itajaí - UNIVALI - Itajaí (SC) - Brasil \\ Petiane Nunes \\ Secretaria de Saúde do Município Balneário Piçarras - Balneário Piçarras (SC) - Brasil
}

\section{RESUMO}

Objetivo: Relatar a experiência de capacitação dos profissionais das equipes de Estratégia Saúde da Família (ESF) da cidade de Balneário Piçarras/SC como estratégia para operacionalizar o SISVAN. Síntese dos dados: Participaram do estudo 67 profissionais das sete equipes de ESF do município, no período de abril a setembro de 2016. Foram realizados dois encontros, um para identificação das dificuldades de implementação do sistema (em que se encontraram os seguintes obstáculos: a multiplicidade de atividades falta de formulários impressos e balanças antropométricas em más condições de uso) e início da capacitação (abordando a importância do SISVAN); e outro para orientar quanto à completude dos formulários e a digitação dos dados no programa. Conclusão: Ao final, observou-se o interesse dos profissionais em tornar o SISVAN ativo. Ainda foi relatada pelos participantes a importância da inserção do profissional de nutrição nas equipes de ESF, colaborando nas atividades referentes à educação alimentar e nutricional, bem como nas orientações e intervenções necessárias à situação alimentar e nutricional da população. Finalizada a capacitação, constatou-se, por meio do sistema, o registro de dados do SISVAN mais eficiente por algumas equipes.

Descritores: Vigilância Nutricional; Capacitação em Serviço; Atenção Primária à Saúde; Estratégia Saúde da Família.

\section{ABSTRACT}

Objective: To report the experience of training Family Health Strategy (Estratégia Saude da Familia - ESF) professionals in the city of Balneário Piçarras, Santa Catarina, as a strategy to operationalize SISVAN. Data synthesis: Participants were 67 professionals from the 7 ESF teams working in the municipality from April to September 2016. Two meetings were held: one for the identification of the difficulties in implementing the system (such as: multiple activities, lack of printed forms, and the poor conditions of anthropometric scales) and initiation of the training (addressing the importance of SISVAN); and the other to teach how to complete the forms and insert the data into the software. Conclusion: After all, the professionals became interested in activating SISVAN. The participants also reported the importance of including nutrition professionals in the ESF teams to collaborate in the activities related to food and nutrition education and in the provision of guidelines and interventions based on the food and nutritional situation of the population. After the training was completed, there was a more efficient data logging into SISVAN by some teams.

Descriptors: Nutritional Surveillance; Inservice Training; Primary Health Care; Family Health Strategy. 


\section{RESUMEN}

Objetivo: Relatar la experiencia de capacitación de profesionales de los equipos de Estrategia Salud de la Familia (ESF) de la ciudad de Balneário Piçarras/SC como estrategia de operacionalización del SISVAN. Síntesis de los datos: Participaron del estudio 67 profesionales de los siete equipos de ESF del municipio en el periodo entre abril y septiembre de 2016. Fueron realizados dos encuentros siendo uno para identificar las dificultades de la implementación del sistema (en el cual se ha encontrado los siguientes obstáculos: la multiplicidad de actividades, la ausencia de formularios impresos y básculas antropométricas con malas condiciones de uso) e inicio de la capacitación (abordando la importancia del SISVAN); y otro para orientar sobre cómo rellenar los formularios y el tecleo de los datos en el programa. Conclusión: Al final se observó el interés de los profesionales en poner el SISVAN activo. También fue relatada por los participantes la importancia de la inserción del profesional de nutrición en los equipos de ESF para colaborar en las actividades de educación alimentaria y nutricional así como en las orientaciones e intervenciones necesarias a situación alimentaria y nutricional de la población. Al final de la capacitación se constató a través del sistema el registro de datos del SISVAN más eficiente de parte de algunos equipos.

Descriptores: Vigilancia Nutricional; Capacitación en Servicio; Atención Primaria de Salud; Estrategia de Salud Familiar.

\section{INTRODUÇÃO}

A avaliação do estado nutricional da população é atitude essencial ao aperfeiçoamento da assistência e da promoção à saúde e necessita ser valorizada ${ }^{(1)}$. No Brasil, o Sistema de Vigilância Alimentar e Nutricional (SISVAN) é utilizado para o monitoramento do padrão alimentar e do estado nutricional dos indivíduos que frequentam as Unidades Básicas de Saúde (UBS), o qual é realizado pelos profissionais das equipes de Estratégia Saúde da Família (ESF) através dos equipamentos antropométricos e formulários específicos impressos. Após a coleta, os dados são digitados no SISVAN Web, que é o sistema informatizado, possibilitando conhecer a situação alimentar e nutricional, e propor melhorias aos problemas prevalentes na população(2).

Desse modo, as equipes de ESF são consideradas elementos fundamentais para apoiar a realização da Vigilância Alimentar e Nutricional ${ }^{(3)}$. Contudo, estudos mostram que diversos problemas na atenção básica dificultam a implementação do SISVAN nos municípios brasileiros, como a falta de equipamentos antropométricos, a sobrecarga de trabalho dos profissionais, internet lenta, falta de profissionais para a digitação dos dados, alta rotatividade de profissionais e a insuficiência de nutricionistas na operacionalização das atividades ${ }^{(4,5)}$. Esses problemas acabam por desmotivar os profissionais das equipes na realização das suas atividades, assim, estudos sugerem a capacitação como uma estratégia para motivá-los a trabalhar com o SISVAN ${ }^{(4,6)}$.

Neste contexto, este trabalho tem como objetivo relatar a experiência de capacitação dos profissionais das equipes de Estratégia Saúde da Família da cidade de Balneário Piçarras/SC como estratégia para operacionalizar o SISVAN.

\section{SÍNTESE DOS DADOS}

A experiência de sensibilização para operacionalizar o SISVAN ocorreu em dois encontros, realizados no período de abril a setembro de 2016 e em todas as Unidades Básicas de Saúde do município de Balneário Piçarras/SC. A cidade está situada na mesorregião do Vale do Itajaí e possui uma área territorial de $99,424 \mathrm{~km}^{2}$. Segundo estimativas do $\mathrm{IBGE}^{(7)}$, o município contava com uma população de 21.253 habitantes em 2016. Na área da saúde, possui seis Unidades Básicas de Saúde e sete equipes da Estratégia Saúde da Família (ESF), constituídas por médicos, enfermeiros, técnicos em enfermagem e agentes comunitários de saúde.

O trabalho envolveu os profissionais de todas as equipes de ESF do município, no total de 67 pessoas. Os encontros foram conduzidos por uma acadêmica do curso de Nutrição da Universidade do Vale do Itajaí sob a orientação da professora envolvida no projeto e participação da nutricionista integrante do Núcleo de Apoio à Saúde da Família (NASF) de Balneário Piçarras.

Este trabalho atendeu às premissas da Resolução n ${ }^{\circ} 466 / 12$ do Conselho Nacional de Saúde e foi aprovado pelo Comitê de Ética em Pesquisa da Universidade do Vale do Itajá sob o Parecer n ${ }^{\circ} 1.355 .933$.

O primeiro encontro ocorreu durante os dois primeiros meses, abril e maio, no qual se realizou uma roda de conversa por unidade para apresentação do projeto e assinatura do Termo de Consentimento Livre e Esclarecido, onde estavam descritas todas as etapas do estudo. Em seguida, foi apresentado um vídeo didático ${ }^{(8)}$ abordando o histórico da construção do SISVAN e destacando a importância dos dados desse sistema para monitorar a situação alimentar e nutricional da população e, a partir disso, propor melhorias aos problemas prevalentes na comunidade. Nesse momento, os participantes esclareceram suas dúvidas sobre o funcionamento do sistema e ainda contribuíram com sugestões para a sua incorporação na rotina das equipes, o que foi de grande relevância, pois as capacitações devem representar uma troca de experiências, na qual, além de transmitir informações e reforçar atitudes positivas, seja possível conhecer a diversidade de vivências e dificuldades enfrentadas pela equipe em sua rotina, bem como suas dúvidas, opiniões e sugestões para melhorar o serviço ${ }^{(9,10)}$. 
Dessa forma, ainda no primeiro encontro, os profissionais foram questionados sobre as principais dificuldades para a implementação do SISVAN e relataram que priorizam as atividades consideradas mais importantes, as quais são constantemente cobradas pela coordenação da unidade, como as atividades assistenciais: os procedimentos médicos e de enfermagem, que correspondem às consultas, aferição de pressão arterial sistêmica, vacinação, dispensação de medicamentos e outras. Percebeuse que as equipes sentem-se desmotivadas a preencher os formulários do SISVAN devido à inexistência do retorno de relatórios com os resultados, além da falta de digitadores e do pouco tempo para a coleta dos dados, ocasionada pela multiplicidade de atividades na UBS, pela falta de formulários impressos e balanças antropométricas em más condições de uso. Durante as visitas, verificou-se que todas as UBS possuem computadores com acesso à internet em bom funcionamento, possibilitando a digitação dos dados no SISVAN Web na própria unidade.

Problemas semelhantes foram encontrados em um estudo que avaliou o uso do SISVAN como instrumento de informação para o planejamento, gestão e avaliação de ações de alimentação e nutrição em municípios de Minas Gerais, sendo a sobrecarga de trabalho, a insuficiência e falta de manutenção dos equipamentos antropométricos, a falta de profissionais para a digitação dos dados e a internet lenta foram algumas das dificuldades relatadas pelos funcionários das unidades ${ }^{(5)}$.

Outro problema relatado pelas equipes é a falta do formulário do SISVAN impresso referente aos marcadores do consumo alimentar. Diferentes estudos corroboram com esses resultados ${ }^{(6,11,12)}$. Uma pesquisa que descreveu a utilização e a cobertura do SISVAN em municípios do Rio Grande do Sul também constatou que a coleta e digitação de dados antropométricos têm sido preponderantes $^{(12)}$.

Desse modo, é recomendado que a avaliação do consumo alimentar seja realizada na rotina dos serviços de Atenção Básica, pois isso permite a observação de comportamento ou padrão que caracteriza marcadores positivos e/ou negativos da alimentação. No SISVAN são avaliados os alimentos consumidos no dia anterior à entrevista por meio de três tipos de formulários: um destinado às crianças menores de 6 meses, outro para crianças de 6 a 23 meses e um para indivíduos com 2 anos ou mais ${ }^{(13)}$.

Quando questionados se a coleta dos dados antropométricos de beneficiários do Programa Bolsa Família (PBF) é priorizada com relação a outros usuários da atenção básica, os profissionais responderam que sim. Tal caso assemelha-se a uma pesquisa que analisou o SISVAN como instrumento de monitoramento da Estratégia Nacional para Alimentação Complementar Saudável (ENPACS) em 38 municípios de Minas Gerais, em que mais de 70\% informaram a priorização da coleta e digitação de dados de indivíduos beneficiários do $\mathrm{PBF}^{(11)}$. É provável que a preferência por estes dados deva-se ao fato de o acompanhamento semestral do estado nutricional das crianças serem uma das condicionalidades de saúde desse programa, uma vez que são compromissos que devem ser cumpridos para que a família continue recebendo o benefício ${ }^{(14)}$. Entretanto, as equipes informaram que tanto essa população quanto os outros usuários da atenção básica apresentam resistência para comparecer à UBS e realizar o acompanhamento nutricional, assim muitas acabam tendo seu benefício suspenso.

O segundo encontro foi planejado considerando as dificuldades apontadas pelas equipes na execução do programa. Elaboraram-se alternativas para incorporar o SISVAN na rotina de suas atividades, juntamente com um treinamento para o preenchimento das fichas do SISVAN e para a digitação dos dados no sistema.

$\mathrm{Na}$ segunda visita às unidades se definiu a população que seria registrada no SISVAN, pois o sistema permite o acompanhamento de indivíduos em todas as fases do curso da vida (crianças, adolescentes, adultos, idosos e gestantes), no entanto, o município pode optar por monitorar somente determinados grupos ${ }^{(2)}$. Assim, a enfermeira coordenadora da Atenção Básica da Secretaria Municipal de Saúde (SMS), que também coordena o SISVAN no município, escolheu acompanhar crianças e gestantes considerando serem os grupos mais vulneráveis diante da realidade de saúde da comunidade assistida. Dessa forma, foi apresentado às equipes o calendário mínimo de consultas para acompanhamento da criança, sugerido pelo Ministério da Saúde, que recomenda o registro do estado nutricional e dos marcadores do consumo alimentar de crianças aos 15 dias de vida, 1 mês, 2, 4, 6, 9, 12, 18 e 24 meses. A partir dos 2 anos, orienta-se a realização de, no mínimo, um registro por ano (acompanhamento nutricional e dos marcadores do consumo alimentar) até que a criança complete 10 anos. Para as gestantes, recomenda-se a realização de, no mínimo, um registro durante a gestação do acompanhamento nutricional e dos marcadores do consumo alimentar ${ }^{(15)}$.

Por conseguinte, foram apresentadas algumas estratégias para coleta de dados que podem ser utilizadas na UBS para realizar a Vigilância Alimentar e Nutricional dos dois grupos, como: durante as consultas de pré-natal e de puericultura, na vacinação, nas ações no âmbito do Programa Saúde na Escola, e na atuação do Agente Comunitário de Saúde, onde foi destacada sua importância na saúde da família, pois é a ponte entre o serviço de saúde e o usuário, e mantém o fluxo contrário entre a população e a informação de saúde ${ }^{(16)}$, podendo atuar na sensibilização das famílias no que se refere à necessidade de comparecer na UBS para realizar o acompanhamento nutricional.

Os profissionais também receberam orientação quanto ao preenchimento correto dos formulários do SISVAN para a obtenção de dados fidedignos. Nesse momento, foi proposto que a digitação dos dados no SISVAN Web aconteça na própria UBS, em razão da ausência de digitadores na SMS. Para tanto, os enfermeiros foram orientados quanto aos procedimentos que devem ser observados ao alimentar o sistema, acessando os formulários do SISVAN e gerando relatórios sobre o estado nutricional da população que acompanham. O objetivo da proposta é tornar as UBS autônomas, de maneira que a própria equipe 
coleta e digita os dados e ainda tem acesso aos resultados, o que pode motivá-las a dar continuidade a essa atividade. Discutiuse que a digitação poderá ser mensal e a equipe poderá revezar este trabalho a partir da organização interna.

$\mathrm{Na}$ oportunidade, os enfermeiros relataram a importância da inserção do profissional de nutrição nas equipes de ESF, colaborando nas atividades referentes à educação nutricional, bem como nas orientações e intervenções necessárias à situação alimentar e nutricional da população. Dessa forma, considera-se que a atuação do nutricionista na atenção básica, especificamente na equipe de ESF, é necessária para a resolução de problemas alimentares e prevenção de doenças causadas pela insegurança alimentar(17).

Como limitações da prática realizada citam-se dificuldade para sistematizar os encontros com as equipes para realização desse trabalho em função das diferentes demandas de atividades nas UBS; dificuldade em operacionalizar sistemas de informática, que se deve, em parte, ao fato de algumas pessoas não estarem incluídas digitalmente; e a alta rotatividade de profissionais nas equipes.

\section{CONCLUSÃO}

Ao final do trabalho proposto, observou-se o interesse dos profissionais em tornar o SISVAN ativo, mesmo com as diversas atividades desenvolvidas na unidade. Tal fato foi relatado também pela nutricionista do serviço, pois depois de finalizada a capacitação, constatou o registro de dados do SISVAN mais eficiente por algumas equipes.

Sugere-se maior organização no processo de trabalho dos grupos em função das atividades serem distribuídas entre a prática, junto à comunidade, e as atividades administrativas desenvolvidas internamente, pois, dessa forma, será possível contemplar a realização de todas as atividades, incluindo a Vigilância Alimentar e Nutricional.

Recomenda-se que o processo de atualização e capacitação das equipes de ESF seja intensificado e contínuo em razão da alta rotatividade de profissionais. Para isso, é essencial que sejam adotadas estratégias dialógicas nessas ações, de modo que os profissionais sintam-se ouvidos e valorizados ao compartilhar suas experiências, problemas e sugestões. Só um processo permanente de capacitação pode proporcionar a adesão dos profissionais na realização de atividades, bem como na continuidade do SISVAN.

\section{REFERÊNCIAS}

1. Ministério da Saúde (BR), Secretaria de Atenção à Saúde, Departamento de Atenção Básica. Orientações para a coleta e análise de dados antropométricos em serviços de saúde: Norma Técnica do Sistema de Vigilância Alimentar e Nutricional - SISVAN. Brasília: Ministério da Saúde; 2011.

2. Ministério da Saúde (BR), Secretaria de Atenção à Saúde, Departamento de Atenção Básica. Política nacional de alimentação e nutrição. Brasília: Ministério da Saúde; 2012.

3. Pimentel VRM, Sousa MF, Hamann EM, Mendonça AVM. Alimentação e nutrição na Estratégia Saúde da Família em cinco municípios brasileiros. Ciênc Saúde Coletiva. 2014;19(1):49-57.

4. Pereira SMPD, Brito LMH, Palácio MAV, Monteiro MPA. Operacionalização do Sistema de Vigilância Alimentar e Nutricional em Juazeiro do Norte, Ceará. Rev Baiana Saúde Pública. 2012;36(2):577-86.

5. Rolim MD, Lima SML, Barros DC, Andrade CLT. Avaliação do SISVAN na gestão de ações de alimentação e nutrição em Minas Gerais, Brasil. Ciênc Saúde Coletiva. 2015;20(8):2359-69.

6. Alves ICR, Souza TF, Pinho L. Operacionalização do sistema de vigilância alimentar e nutricional: relato de experiência. Rev APS. 2015;18(3):398-402.

7. Instituto Brasileiro de Geografia e Estatística - IBGE. Cidades: Balneário Piçarras [acesso em 2016 Nov 9]. 2016. Disponível em: http://www.cidades.ibge.gov.br/xtras/perfil.php?lang=\&codmun=4212809

8. Sautter L. Trabalho de nutrição em saúde pública. 2014. 1 vídeo. Online [acesso em 2016 Abr 1]. Disponível em: https:// www.youtube.com/watch?v=AE_AEdvxB4

9. Salas E, Diaz Granados D, Weaver SJ, King H. Does team training work? Principles for health care. Acad Emerg Med. 2008;15(11):1002-9.

10. Bagni UV, Barros DC. Capacitação em antropometria como base para o fortalecimento do Sistema de Vigilância Alimentar e Nutricional no Brasil. Rev Nutr. 2012;25(3):393-402.

11. Ferreira CS, Cherchiglia ML, César CC. O Sistema de Vigilância Alimentar e Nutricional como instrumento de monitoramento da Estratégia Nacional para Alimentação Complementar Saudável. Rev Bras Saúde Matern Infant. 2013;13(2):167-77. 
12. Jung NM, Bairros FS, Neutzling MB. Utilização e cobertura do Sistema de Vigilância Alimentar e Nutricional no Estado do Rio Grande do Sul, Brasil. Ciênc Saúde Coletiva. 2014;19(5):1379-88.

13. Ministério da Saúde (BR), Secretaria de Atenção à Saúde, Departamento de Atenção Básica. Marco de referência da vigilância alimentar e nutricional na atenção básica. Brasília: Ministério da Saúde; 2015.

14. Ministério da Saúde (BR), Secretaria de Atenção à Saúde, Departamento de Atenção Básica. Manual de orientações sobre o Bolsa Família na Saúde. Brasília: Ministério da Saúde; 2010.

15. Ministério da Saúde (BR), Secretaria de Atenção à Saúde, Departamento de Atenção Básica. Protocolos do Sistema de Vigilância Alimentar e Nutricional - SISVAN na assistência à saúde. Brasília: Ministério da Saúde; 2008.

16. Costa SM, Araújo FF, Martins LV, Nobre LLR, Araújo FM, Rodrigues CAQ. Agente Comunitário de Saúde: elemento nuclear das ações em saúde. Ciênc Saúde Coletiva. 2013;18(7):2147-56.

17. Sena JN, Pereira MS, Rodrigues, RB. Reflexões sobre saúde, nutrição e Estratégia Saúde da Família no contexto de integralidade do Sistema Único de Saúde (SUS). Rev Rede Cuidados Saúde. 2015;9(3):1-25.

\section{Endereço primeiro autor:}

Éllen Luzia Soares

Universidade do Vale do Itajaí

Rua Uruguai, 458

Bairro: Centro

CEP 88302-202 - Itajaí - SC - Brasil

E-mail: ellens402@gmail.com

\section{Endereço para correspondência:}

Tatiana Mezadri

Universidade do Vale do Itajaí

Mestrado Profissional em Saúde e Gestão do Trabalho

Rua Uruguai, 458 - Bloco F6 - Terceiro piso

Bairro: Centro

CEP 88302-202 - Itajaí - SC - Brasil

E-mail: mezadri@univali.br 